\title{
Avaliação in vitro do potencial erosivo e cariogênico de antifúngicos tópicos
}

\author{
In vitro evaluation of erosive and cariogenic potential of topical antifungal agents
}

Juliana Borges de Lima Dantas ${ }^{1}$, Elisângela de Jesus Campos², Gabriela Botelho Martins ${ }^{3 *}$

\begin{abstract}
Mestre em Estomatologia. Professora Assistente da Faculdade Adventista da Bahia; ${ }^{2}$ Doutora em Medicina e Saúde. Professora Adjunta do Instituto de Ciências da Saúde da Universidade Federal da Bahia; ${ }^{3}$ Doutora em Estomatologia

Clínica. Professora Adjunta do Instituto de Ciências da Saúde da Universidade Federal da Bahia.
\end{abstract}

\begin{abstract}
Resumo
Introdução: a candidíase é uma infecção fúngica oportunista, causada pela proliferação e disseminação de espécies de Candida, que pode acometer a cavidade oral. Dentre os antifúngicos mais utilizados e de uso tópico, a nistatina é considerada o medicamento de primeira escolha. Objetivo: avaliar as propriedades físico-químicas de diferentes marcas de nistatina disponíveis no mercado, incluindo o pH, a acidez total titulável (ATT) e a determinação de sólidos solúveis totais (SST). Metodologia: trata-se de um estudo experimental in vitro, constituído por uma amostra de oito diferentes marcas de nistatina em suspensão oral de uso tópico. Foi analisado o potencial erosivo e cariogênico dessas soluções mediante a determinação de pH, ATT e SST ( $\left.{ }^{\circ} B r i x\right)$. Resultados: no tocante ao pH, verificou-se que a média obtida foi de $6,05( \pm 0,66)$. Dois dos medicamentos analisados (marcas A e H) apresentaram pH abaixo do crítico para a dissolução do esmalte dental. Quanto à ATT das soluções, os valores variaram de 1,9 a $14,53 \mathrm{~mL}$ para atingir o pH neutro, indicando que as marcas B, C e E podem levar mais tempo para ser neutralizadas em razão da quantidade de solução necessária. A análise do 'Brix revelou que a marca $\mathrm{H}$ apresentou o maior teor de açúcares em sua composição (44,9\%). Conclusão: a formulação de nistatina da marca $\mathrm{H}$ apresentou $\mathrm{pH}$ endógeno mais crítico e percentual de sólidos solúveis totais elevado, sendo, portanto, a medicação com maior fator de risco para o desenvolvimento de cárie e erosão dentária, devendo ser consideradas as doses e frequências de uso, bem como os hábitos de higiene oral do paciente.
\end{abstract}

Palavras-chave: Candidíase Oral. Nistatina. Cárie Dentária. Erosão Dentária.

\begin{abstract}
Introduction: candidiasis is an opportunistic fungal infection caused by the proliferation and spread of Candida species that can affect the oral cavity. Among the most commonly used topical antifungal agents, nystatin is considered the first choice drug. Methodology: to evaluate the physical and chemical properties of different brands of nystatin available in the market, including $\mathrm{pH}$, titratable acidity and determination of total soluble solids. Results: Regarding $\mathrm{pH}$, it was verified that the mean obtained was 6.05 ( \pm 0.66$)$. Two of the analyzed drugs $(A$ and $H$ ) presented $\mathrm{pH}$ below that considered critical for the dissolution of dental enamel. As for the titratable total acidity of the solutions, values ranged from 1.9 to $14.53 \mathrm{~mL}$ to reach neutral $\mathrm{pH}$, indicating that the $B, C$ and $E$ marks may take longer to neutralize because of the amount of solution required. The analysis of ${ }^{\circ}$ Brix revealed that the $\mathrm{H}$ mark had the highest sugar content in its composition (44.9\%). Conclusion: Nystatin brand $\mathrm{H}$ presented the worst indices in terms of endogenous $\mathrm{pH}$ and total sugar percentage, being therefore the medication with the highest risk factor for the development of caries and dental erosion. Keywords: Oral candidiasis. Nystatin. Dental caries. Tooth erosion.
\end{abstract}

\section{INTRODUÇÃO}

A cavidade oral constitui o habitat de uma microbiota diversificada que, em condições alteradas, favorece o surgimento de infecções oportunistas (MEURMAN et al., 1997). Uma complexa interação de fatores contribui para o surgimento de infecções, em especial da candidíase, a exemplo da mucosite oral radio ou quimioinduzida, hipossalivação e comprometimento do sistema imunológico (FISCHER; EPSTEIN, 2008).

A candidíase é uma infecção fúngica oportunista causada pela proliferação e disseminação de espécies de Candida - especialmente a C. albicans -, que são

Correspondente/Correspondente: *Gabriela Botelho Martins - End: Av. Reitor Miguel Calmon S/N sala 413 Canela Salvador-Ba CEP: 40231-300 - Tel: (71) 3283-8891 - E-mail: gbmartinsba@gmail.com habitantes comensais da cavidade oral. Essa infecção representa um dos efeitos mais frequentes e perturbadores em pacientes oncológicos, pois afeta diretamente sua qualidade de vida. Além de acometer grande parte dos pacientes imunossuprimidos, tem também predileção por idades extremas, apresentando altas taxas de ocorrência em crianças e idosos (GORDÓN-NÚÑEZ; PINTO, 2003).

O tratamento dessa condição pode ser realizado com o uso de medicações tópicas ou sistêmicas. Em geral, é preferível a utilização de antifúngicos locais, pois eles apresentam menor risco de efeitos colaterais e de interações medicamentosas (LALLA et al., 2010). Dentre os antifúngicos mais utilizados, de uso tópico, a nistatina é considerada o medicamento de primeira escolha para o tratamento da candidíase orofaríngea leve, de acordo com a Sociedade de Doenças Infecciosas da América, IDSA 
(PAPPAS et al., 2009). Todavia informações sobre o pH, a acidez total titulável (ATT) e os sólidos solúveis totais (SST) presentes nos medicamentos que contêm esse fármaco não foram amplamente avaliados na literatura, o que gera um questionamento sobre seu possível potencial cariogênico e erosivo na cavidade oral, principalmente em pacientes oncológicos, que fazem uso constante dessa modalidade terapêutica, além do estado de imunossupressão e hipossalivação associados à terapia oncológica, o que predispõe à atividade cariogênica local.

Dessa maneira, face à existência de poucos estudos sobre esse tema, o objetivo do presente trabalho foi avaliar as propriedades físico-químicas de diferentes marcas de formulações do tipo suspensão oral de nistatina disponíveis no mercado, incluindo o $\mathrm{pH}$, a ATT e a determinação de STT ('Brix), na tentativa de obter dados que revelem a sua atividade local, no que se refere ao potencial erosivo e cariogênico. Esses dados poderão auxiliar a minimizar possíveis efeitos deletérios causados por tais medicações, o que pode ser crucial à saúde bucal dos pacientes.

\section{MATERIAIS E MÉTODOS}

Trata-se de um estudo experimental in vitro, conduzido no Laboratório de Bioquímica Oral do Instituto de Ciências da Saúde (ICS) da Universidade Federal da Bahia (UFBA). A amostra foi composta por oito diferentes marcas de medicamentos do tipo nistatina oral de uso tópico (Quadro 1), um antifúngico amplamente utilizado na Odontologia. Sua apresentação se encontra sob a forma de suspensão oral (100.000 UI / ml), com indicação de uso que pode variar de 1 a $6 \mathrm{~mL}, 4$ vezes ao dia, conforme a orientação dos fabricantes. A determinação das propriedades físico-químicas tais como $\mathrm{pH}$, ATT e STT ( ${ }^{\circ} \mathrm{Brix}$ ) foi realizada em triplicata e sob temperatura local controlada a $25^{\circ} \mathrm{C}$. A média das três amostras de lotes diferentes foram registradas em ficha específica, sendo os dados coletados por um único examinador previamente preparado.

\section{Determinação do potencial hidrogeniônico $(\mathrm{pH})$ das soluções}

A verificação dos valores de $\mathrm{pH}$ foi feita utilizando-se o pHmetro digital Q400-A (Quimis ${ }^{\circledR}$ Aparelhos Científicos Ltda., São Paulo, Diadema, Brasil), combinado com o agitador magnético EV2.017 (EVLAB, Curitiba, Paraná, Brasil). Após a calibração prévia do aparelho, conforme orientação do fabricante, com as soluções tampão de $\mathrm{pH} 7$ e pH 4, foram transferidos $50 \mathrm{~mL}$ de cada medicamento para um becker e, em seguida, foram feitos a leitura e o registro do valor do $\mathrm{pH}$ em ficha específica.

\section{Determinação da acidez titulável das soluções (ATT)}

A ATT dos medicamentos foi determinada de acordo com o método adotado pela Associação Analítica de Oficiais Químicos (1984), adicionando-se, gradativamente, a essas soluções, volumes de hidróxido de sódio $(\mathrm{NaOH})$ a $0,1 \mathrm{~N}$, utilizando-se uma bureta de $50 \mathrm{~mL}$ e sob agitação constante. Os volumes de solução de $\mathrm{NaOH}$ necessários para atingir o pH de 5,5 e de 7 foram registrados, o que correspondeu ao valor da acidez titulável de cada suspensão de nistatina para alcançar os níveis de $\mathrm{pH}$ crítico para dissolução do esmalte dentário e o pH neutro, respectivamente.

\section{Determinação dos sólidos solúveis totais (SST) ('Brix)}

'Brix é uma escala numérica que mede a quantidade de SST em uma solução. Em muitos casos, todo o soluto dissolvido é o açúcar. A determinação dos SST para avaliação da concentração em carboidratos foi realizada por refratometria, método físico, simples e rápido, utilizando refratômetro modelo HI 96801 (Hanna ${ }^{\circledR}$, Woonsocket, Rhodeisland, EUA), calibrado com água destilada a $25^{\circ} \mathrm{C}$.

Análise estatística

Os dados foram organizados no programa Microsoft Excel 2007 e apresentados por meio de estatística descritiva: médias, desvio-padrão e coeficiente de variância.

Quadro 1 - Marcas de nistatina (suspensão oral $100.000 \mathrm{UI} / \mathrm{mL}$ ) comercialmente disponíveis, seus componentes descritos nas bulas e lotes analisados.

\begin{tabular}{|l|l|l|}
\hline $\begin{array}{l}\text { MARCAS NISTATINA } \\
\text { UI/mL }\end{array}$ & & \multicolumn{1}{|c|}{ INGREDIENTES } \\
\hline $\begin{array}{l}\text { TEUTO } \\
\text { Marca A }\end{array}$ & $\begin{array}{l}\text { Água de osmose reversa, carmelose sódica, glicerina, sacarina sódica, ciclamato de sódio, álcool } \\
\text { etílico, metilparabeno, metabissulfito de sódio, propilparabeno, hidróxido de sódio, aroma de menta } \\
\text { e aroma de cereja. }\end{array}$ & $\begin{array}{r}\text { L2643098 } \\
\text { L2643097 } \\
\text { L1504093 }\end{array}$ \\
\hline $\begin{array}{l}\text { GERMED } \\
\text { Marca B }\end{array}$ & $\begin{array}{l}\text { Hietelose, simeticona, sacarina sódica, propilparabeno, metilparabeno, hidróxido de sódio, glicerol, } \\
\text { fosfato de sódio dibásico, essência de framboesa, edetato dissódico di-hidratado, álcool etílico, ácido } \\
\text { cítrico e água purificada. }\end{array}$ \\
\hline $\begin{array}{l}\text { EMS } \\
\text { Marca C }\end{array}$ & $\begin{array}{l}\text { Hietelose, sacarina sódica, propilparabeno, metilparabeno, hidróxido de sódio, essência de framboesa, } \\
\text { álcool etílico, ácido cítrico, edetato dissódico di-hidratado, fosfato de sódio dibásico, glicerol, simeticona } \\
\text { e água purificada. }\end{array}$ \\
\hline $\begin{array}{l}\text { NEOQUÍMICA } \\
\text { Marca D }\end{array}$ & $\begin{array}{l}\text { Carmelose, sacarina sódica, propilparabeno, ciclamato de sódio, álcool etílico, metilparabeno, } \\
\text { metabissulfito de sódio, essência de tutti-frutti, glicerol, silicato de alumínio e magnésio, glicerole água. }\end{array}$ \\
\hline $\begin{array}{l}\text { L915997 } \\
\text { L900936 }\end{array}$ \\
\hline
\end{tabular}




\begin{tabular}{|c|c|c|}
\hline $\begin{array}{l}\text { MARCAS NISTATINA } \\
\text { SOLUÇÃO ORAL } 100.000 \\
\text { UI/mL }\end{array}$ & INGREDIENTES & LOTES \\
\hline $\begin{array}{l}\text { NISTAMAX } \\
\text { Marca E }\end{array}$ & $\begin{array}{l}\text { Glicerina, metilparabeno, propilparabeno, álcool extra neutro, essência de framboesa, essência de } \\
\text { menta, edta dissódico di-hidratado, simeticona 30\%, hidroxietilcelulose, hidróxido de sódio, ácido } \\
\text { cítrico anidro, sacarina sódica, água purificada. }\end{array}$ & $\begin{array}{l}\text { L18260 } \\
\text { L18263 } \\
\text { L18249 }\end{array}$ \\
\hline $\begin{array}{l}\text { LEGRAND } \\
\text { Marca F }\end{array}$ & $\begin{array}{l}\text { Hietelose, simeticona, sacarina sódica, propilparabeno, metilparabeno, hidróxido de sódio, glicerol, } \\
\text { fosfato de sódio dibásico, essência de framboesa, edetato dissódico di-hidratado, álcool etílico, ácido } \\
\text { cítrico, água purificada. }\end{array}$ & $\begin{array}{r}\text { LOH7355 } \\
\text { L880187 } \\
\text { LOC0304 }\end{array}$ \\
\hline $\begin{array}{l}\text { NOVA QUÍMICA } \\
\text { Marca G }\end{array}$ & $\begin{array}{l}\text { Hietelose, simeticona, sacarina sódica, propilparabeno, metilparabeno, hidróxido de sódio, glicerol, } \\
\text { fosfato de sódio dibásico, essência de framboesa, edetato dissódico di-hidratado, álcool etílico, ácido } \\
\text { cítrico e água purificada. }\end{array}$ & $\begin{array}{l}\text { L968464 } \\
\text { L900938 } \\
\text { L939467 }\end{array}$ \\
\hline $\begin{array}{l}\text { PRATI, DONADUZZI \& CIA } \\
\text { Marca H }\end{array}$ & $\begin{array}{l}\text { Glicerol, carmelose sódica, sacarose refinada, sacarina sódica di-hidratada, metilparabeno, } \\
\text { propilparabeno, edetato dissódico, fosfato sódico dibásico, butil-hidroxitolueno, aroma de cereja } \\
\text { líquido, aroma de hortelã pimenta, aroma de canela líquido, álcool etílico e água purificada. }\end{array}$ & $\begin{array}{l}\text { L18B319 } \\
\text { L17G15J } \\
\text { L17A344 }\end{array}$ \\
\hline
\end{tabular}

Fonte: Laboratório de Bioquímica Oral, ICS/ UFBA, 2018.

\section{RESULTADOS}

A Tabela 1 apresenta os valores médios para o pH endógeno, ATT e STT ('Brix) das diferentes amostras de nistatina suspensão oral analisadas.

Tabela 1 - Valores médios para o pH endógeno, acidez titulável e sólidos solúveis totais ( ${ }^{\circ}$ Brix) das suspensões de nistatina oral.

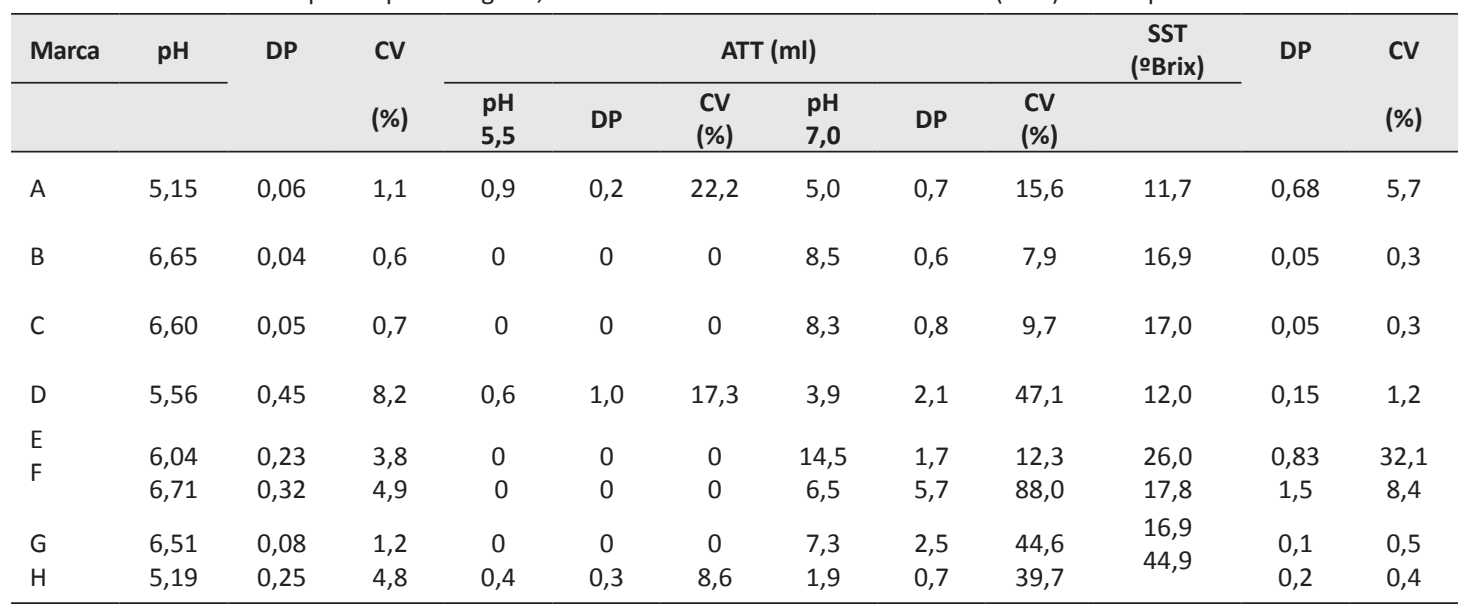

*DP - Desvio-padrão

$* * \mathrm{CV}$ - Coeficiente de variação

Fonte: Laboratório de Bioquímica Oral, ICS/ UFBA, 2018.

No tocante ao $\mathrm{pH}$, verificou-se que a média obtida foi de $6,05( \pm 0,66)$. Dois dos medicamentos analisados (25\%) apresentaram $\mathrm{pH}$ abaixo do considerado crítico para a dissolução do esmalte dental ( $\mathrm{pH}$ 5,5), com valores de 5,15 para a marca A e 5,19 para a H (Tabela 1). Além de apresentar o segundo $\mathrm{pH}$ mais baixo, a marca $\mathrm{H}$ apresentou o maior coeficiente de variação $(4,8 \%)$ em relação às outras marcas analisadas. Os valores de $\mathrm{pH}$ apresentaram uma variação que oscilou de 5,15 (marca A) para 6,71 (marca F), conforme está demonstrado na Figura 1.

Quanto à ATT das soluções, os valores variaram de 1,9 a $14,5 \mathrm{ml}$ de solução de $\mathrm{NaOH} 0,1 \mathrm{~N}$ para atingir o $\mathrm{pH}$ neutro $(7,0)$, indicando que alguns dos medicamentos, incluindo as marcas $B, C$ e $E$, podem levar mais tempo para ser neutralizados em razão da quantidade de solução necessária. As marcas $\mathrm{H}$ e E apresentaram os menores e os maiores valores de ATT, respectivamente (Tabela 1).
Figura 1 - Distribuição das soluções de nistatina segundo o pH (média).

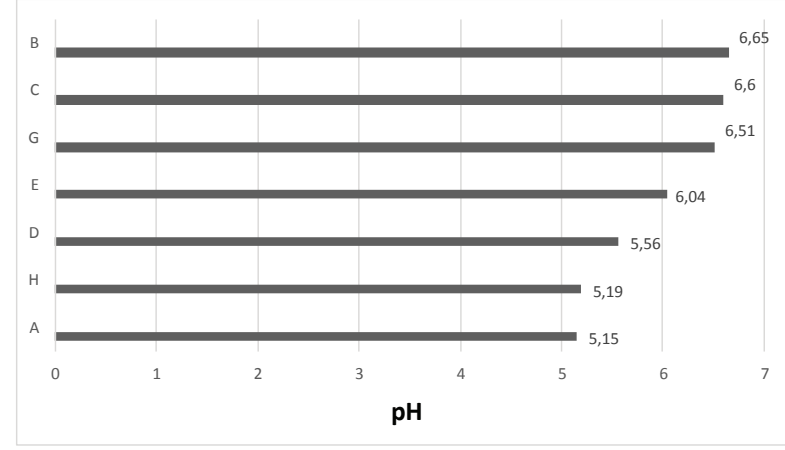

Fonte: Laboratório de Bioquímica Oral, ICS/ UFBA, 2018. 
A análise do ${ }^{\circ}$ Brix revelou que a maioria das formulações com nistatina (75\%) apresentou valores inferiores a $20 \%$ (Tabela 1). Das soluções testadas, a marca H apresentou o maior (44,9\%) e a marca A apresentou o menor valor de SST, com 11,7\% (Figura 2).

Figura 2 - Distribuição das suspensões de nistatina segundo o teor de sólidos solúveis totais.

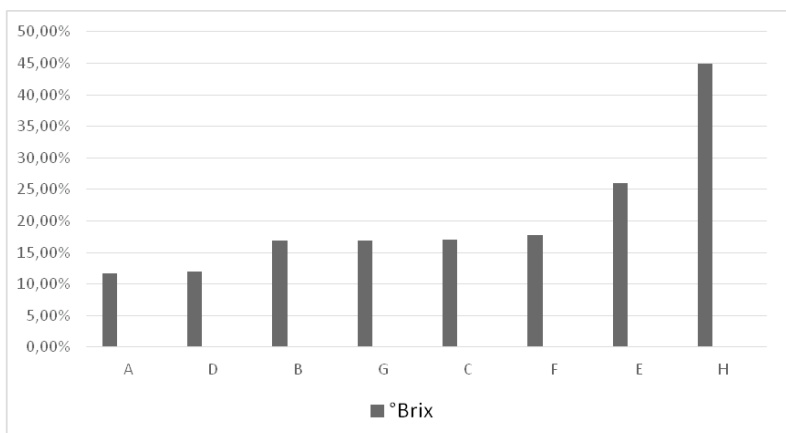

Fonte: Laboratório de Bioquímica Oral, ICS/ UFBA, 2018.

\section{DISCUSSÃO}

O uso de medicamentos faz parte do cotidiano de muitas pessoas que sofrem, principalmente, de problemas crônicos, e, por isso, são considerados componentes imprescindíveis à melhoria do bem-estar físico e mental dos indivíduos. Muitas dessas medicações apresentam, em sua composição, substâncias que podem prejudicar a saúde bucal dos pacientes, e, portanto, causar a cárie e a erosão dentária (MARQUEZAN et al., 2006). Devido à alta frequência de uso da nistatina na forma de suspensão oral por grupos distintos de pacientes, faz-se necessário o estudo de suas propriedades físico-químicas, uma vez que existe escassez de trabalhos acerca desse tema. Além disso, tal estudo pode propiciar uma orientação adequada a pacientes portadores de candidíase oral sobre os possíveis danos locais e as formas de minimizá-los.

No presente estudo, dois dos oito medicamentos analisados obtiveram valores médios de $\mathrm{pH}$ abaixo do valor crítico para a dissolução do esmalte $(\mathrm{pH}=5,5)$. As marcas de suspensões de nistatina que apresentaram os níveis de $\mathrm{pH}$ mais baixos foram $\mathrm{A}$ e $\mathrm{H}, \mathrm{com} \mathrm{pH}=5,15$ e 5,19 , respectivamente. A marca $D$ obteve um $\mathrm{pH}$ médio de 5,56, e, apesar de estar acima do valor crítico, seu coeficiente de variância foi alto $(8,2 \%)$. Estudos prévios demonstraram que inúmeras classes de medicamentos apresentam valores de $\mathrm{pH}$ abaixo do considerado crítico para dissolução do esmalte dentário (CAVALCANTI et al., 2012; SOUSA et al., 2010). Entretanto, na literatura, existem poucos estudos que avaliaram o $\mathrm{pH}$ de antifúngicos orais (VERMERIE et al., 1997). Os valores de pH abaixo do neutro encontrados no presente estudo, podem estar relacionados com a presença de ácidos nas formulações dos fármacos, a exemplo do ácido cítrico, que representa o principal tipo de ácido orgânico fraco utilizado como conservante na maioria dos medicamentos, e que pode produzir altos níveis de erosão em virtude da suas propriedades quelantes (SOUSA et al., 2010). De acordo com as bulas, o ácido cítrico foi encontrado em 5 (62,5\%), das 8 formulações analisadas, embora as duas formulações que apresentaram os menores valores de $\mathrm{pH}(\mathrm{A}$ e $\mathrm{H}$ ) não contivessem esse tipo de ácido em sua composição, e sim o álcool etílico. Segundo Moreira (2011), o ácido cítrico é utilizado para redução do $\mathrm{pH}$, controle de crescimento microbiano, aromatizante para mascarar gosto desgradável da sacarina e para ação quelante.

$\mathrm{O} \mathrm{pH}$ das medicações líquidas geralmente está relacionado com a estabilidade da substância química, em virtude da possível decomposição em função do $\mathrm{pH}$ inadequado, o que compromete diretamente sua ação farmacológica (FERREIRA; SOUZA, 2005). Os intervalos de valores de $\mathrm{pH}$ recomendados para estabilizar as suspensões de nistatina sem interferir na eficácia antifúngica são de 5,0 a 7,0 ou de 6,0 a 8,0, (HAMILTON-MILLER, 1973). No estudo realizado por Vermerie et al. (1997), o solvente alcalino adicionado na solução de Mycostatin TM (Bristol-Myers Squibb ${ }^{\circledR}$, Paris, France), com pH 7,9, não alterou a estabilidade da nistatina em comparação com a suspensão aquosa (pH 5,4). De acordo com esses resultados, pode-se concluir que todas marcas analisadas no presente estudo apresentam estabilidade e eficácia antifúngica, pois os valores de pH variaram de 5,15 a 6,71.

$\mathrm{Na}$ comparação com outros estudos realizados com diferentes classes de medicamentos utilizados em pacientes pediátricos, pôde-se verificar uma média de $\mathrm{pH}$ mais elevada no presente estudo, indicando que a classe de antibióticos, anti-histamínicos e corticosteroides geralmente necessitam de um $\mathrm{pH}$ menor para manter sua estabilidade e função (CAVALCANTI et al., 2012; SOUSA et al., 2010). No entanto, por mais que a maioria das formulações de nistatina tenha apresentado valores médios de $\mathrm{pH}$ superiores a 5,5, deve-se ressaltar que todas as suspensões apresentaram médias inferiores a 7,0, e, portanto, são ácidas. A análise individual de cada medicamento considerado nesta investigação mostrou resultados que variaram de $\geq 5,15 \mathrm{a} \geq 6,71$, fato que possivelmente pode favorecer a ocorrência do processo de desmineralização, a depender da dose e da frequência do uso desses medicamentos.

$\mathrm{O} \mathrm{pH}$ endógeno de um medicamento pode ser rapidamente modificado na cavidade oral pela ação neutralizadora da saliva. No entanto, vários medicamentos podem demorar mais tempo para ser tamponados pela saliva, pois, para isso, é necessário um maior volume de bicarbonato salivar. Além disso, algumas condições inerentes ao indivíduo podem acarretar também a demora do tamponamento salivar, a exemplo dos pacientes imunossuprimidos e aqueles que, por algum motivo, apresentam modificação qualitativa e quantitativa da saliva. A acidez de uma solução pode ser expressa em termos de ATT, que é uma medida do peso percentual de ácido presente em uma solução, calculada a partir do volume de sódio hidróxido $(\mathrm{NaOH})$ ou hidróxido de potássio 
(KOH) necessário para neutralizar essas espécies ácidas (CAVALCANTI et al., 2012).

Sabe-se que algumas medicações, principalmente na forma de xaropes, podem provocar uma queda do $\mathrm{pH}$ do biofilme para um nível crítico (abaixo de 5,5) em poucos minutos, iniciando o processo de desmineralização do esmalte dentário. De forma subsequente, inicia-se a restituição do $\mathrm{pH}$ da saliva, com a finalidade de restabelecer o equilíbrio através da remineralização. No entanto, quanto maior for a viscosidade de uma preparação ingerida, o retorno ao $\mathrm{pH}$ neutro é considerado bastante lento (SANTINHO; WALDOW; SANTOS, 2008). No presente estudo, foi observado que as marcas $\mathrm{B}, \mathrm{Ce}$ E necessitaram de maiores volumes de solução de $\mathrm{NaOH}$ a 0,1 N para atingir o pH 7 , o que sugere, assim, ter havido, nessas marcas, maior resistência para elevar o $\mathrm{pH}$ à neutralidade, o que favorece o processo de desmineralização. Adicionalmente, não se podem negligenciar os fatores inerentes ao paciente, no que diz respeito a seu estado de saúde geral e à manutenção de uma higiene bucal rigorosa, o que pode atenuar e até prevenir essa complicação.

Além dessas interações químicas entre os dentes e os fluidos bucais, outras condições podem ter um impacto sobre a erosão dentária in vivo, como os fatores biológicos, que incluem a hipossalivação, a capacidade tampão e a composição salivar. Esses fatores devem ser levados em consideração, principalmente quando se trata de pacientes enfermos, a exemplo dos diabéticos, hipertensos e aqueles submetidos à terapia oncológica, uma vez que haverá uma mudança sistêmica e local nesses indivíduos, o que acarreta possível mudança da microflora bucal, devido ao uso de medicamentos que propiciam essa condição (REDONDO; MONTOYA, 2012).

O uso frequente e contínuo de medicamentos é um fator que pode contribuir não só para seu potencial erosivo, como também cariogênico. Assim, o presente estudo selecionou para avaliação in vitro diferentes marcas de nistatina na forma de suspensão oral, uma vez que seu uso é amplamente difundido em crianças, idosos e pacientes imunossuprimidos. Dessa maneira, torna-se importante o estudo dessa substância tendo em vista a possibilidade de risco elevado no desenvolvimento de cárie e erosão dentária. Embora esses dois processos sejam diferentes, as duas condições podem ocorrer simultaneamente e gerar danos aos tecidos duros dentais (CAVALCANTI et al., 2012). De acordo com Phillips e Dellinger (2005), alguns medicamentos sob a forma de solução para bochecho, a exemplo da nistatina, contêm açúcar, o que leva ao aumento do risco de cárie, particularmente em pacientes com hipossalivação. De fato, no presente estudo, pôde ser encontrado, na da marca $\mathrm{H}$, um teor elevado de SST $(44,9 \%)$, incluindo a sacarose entre eles. No entanto, esse potencial cariogênico pode ser reduzido mediante controle da dose e da frequência de uso da medicação, além da ação de uma higiene bucal rigorosa.

Algumas doenças ou medicamentos podem aumentar o risco de surgimento ou a severidade da cárie dentária. 0 paciente portador de uma enfermidade vive uma situação peculiar específica, na qual fatores cariogênicos podem prevalecer (MARQUEZAN et al., 2006). No entanto, o risco maior pode estar relacionado ao tratamento da doença, pois vários medicamentos orais líquidos apresentam baixo $\mathrm{pH}$, alta concentração de açúcares e acidez elevada, o que resulta em potencial cariogênico (DURWARD; THOU, 1997; CAVALCANTI et al., 2012) e erosivo para a estrutura dentária (DURWARD; THOU, 1997).

A análise dos teores de sólidos solúveis totais ('Brix) revelou que o medicamento $\mathrm{H}$ apresentou um teor elevado, com $44,9 \%$, seguido pela marca $\mathrm{E}$, com $26 \%$. Entretanto, ao analisar os componentes dessas duas diferentes formulações, pode-se observar que apenas a marca $\mathrm{H}$ apresentou sacarose em sua composição. As demais marcas avaliadas utilizaram substitutos de açucares ou edulcorantes, a exemplo da sacarina sódica e do ciclamato de sódio, conforme mostra o Quadro 1, que não participam do processo de formação da cárie dentária. Sendo assim, verifica-se que apenas a solução de nistatina da marca $\mathrm{H}$ possui potencial cariogênico relacionado à presença de sacarose. Uma possível justificativa para o aumento do SST em marcas que não continham a sacarose em sua composição se deve à presença de aromatizantes e flavorizantes, compostos que podem estar modificando diretamente esse teor. Entretanto, essa possibilidade não pode ser confirmada, uma vez que os seus quantitativos são segredos industriais, ou seja, não vêm especificados nas bulas (BALBANI; STELZER; MONTOVANI, 2006) e os seus constituintes não são descritos. Ao analisar 73 formulações farmacêuticas sob a forma de gotas ou soluções orais, xaropes, suspensões orais e granuladas, um estudo verificou que $83 \%$ dos medicamentos continham aroma de frutas e $22 \%$ apresentavam aromas ou essências de anis, canela, chiclete, chocolate, ovo, cassis, licor, mel, mentol, rum, entre outros (BALBANI; STELZER; MONTOVA$\mathrm{NI}, 2006)$. No presente trabalho, os agentes flavorizantes descritos incluíam aroma de menta, de cereja, de canela e de hortelã-pimenta, além de essência de framboesa e tutti-frutti. Dessa maneira, a interpretação dos valores de SST através de uma correlação direta com os seus constituintes é dificultada.

Outro fator que deve ser levado em consideração é a posologia das medicações, pois ela também tem influência direta na cariogenicidade. Ou seja, quanto maior a frequência diária de uso, maior o número de desafios cariogênicos, pois a cárie dentária é uma doença multifatorial, dieta-dependente, atribuída, primariamente, à presença de bactérias orais e açúcares fermentáveis (MARQUEZAN et al., 2006). Visto que suspensões de nistatina $(100.000 \mathrm{UI} / \mathrm{mL}$ ) são administradas durante um período que pode variar de uma a várias semanas, com recomendação de uso por 4 vezes ao dia, com volume que pode variar de $5 \mathrm{ml}$ até $15 \mathrm{ml}$ e um tempo de uso que pode se estender de 1 a 5 minutos (VERMERIE et al., 1997), esse medicamento pode ser configurado com alto potencial cariogênico devido a seus componentes, principalmente 
no que diz respeito à marca que apresentou altos teores de SST devido à presença de sacarose refinada em sua formulação, um carboidrato fermentável com potente ação cariogênica. Uma justificativa para a presença de sacarose na preparação farmacêutica se deve ao fato de esse açúcar ter um baixo custo e não deixar gosto residual, além de melhorar a viscosidade do medicamento (CAVALCANTI et al., 2012).

De acordo com Aires et al. (2006), o teor de SST necessário para formar o biofilme cariogênico é de $5 \%$. Entretanto, apesar de todas as marcas de nistatina avaliadas no presente estudo terem apresentado índices acima desse valor, seria errôneo afirmar que todas essas medicações apresentam potencial cariogênico. Isso porque, com exceção da marca $\mathrm{H}$, que apresentou capacidade para a produção de polissacarídeos extracelulares, os quais podem ocasionar o desenvolvimento de lesões de cárie dental, as demais marcas substituíram a sacarose pela sacarina sódica, um adoçante artificial que não apresenta poder cariogênico (DUARTE et al., 2008). Atualmente, pesquisas que buscam encontrar um substituto ideal para o açúcar nas medicações líquidas têm observado, no xilitol, uma alternativa promissora.

Devido à importância de todos esses fatores mencionados, faz-se necessária uma maior interação entre os pacientes e os cirurgiões-dentistas, a fim de promover um suporte preventivo adequado a esses pacientes com risco comprometimento da saúde dentária. Tal suporte consiste em esclarecimento em relação aos possíveis danos à estrutura dental, ocasionados pelas formulações que contêm a nistatina, orientando-os sobre a necessidade de medidas preventivas, como uma higiene bucal efetiva, pois o risco de algum dano local se torna ainda maior quando nenhuma medida efetiva de higiene é realizada para eliminar os resíduos dessas substâncias da cavidade bucal.

Face ao exposto, uma limitação encontrada se refere à busca bibliográfica realizada, onde foi encontrado um reduzido número de estudos que avaliaram as propriedades físico-químicas dos medicamentos com nistatina, com diferenças metodológicas consideráveis. Por fim, constituiu uma limitação a falta de acesso à concentração dos constituintes encontrados nas diferentes formulações de nistatina, uma vez que os fabricantes não forneceram esse tipo de informação, impedindo uma análise mais acurada.

\section{CONCLUSÃO}

A candidíase oral é uma condição clínica muito comum, e a prescrição de antifúngicos faz parte da rotina diária do cirurgião-dentista. No presente estudo, observou-se que o potencial erosivo das formulações com nistatina foi baixo onde foram encontrados valores de pH abaixo do crítico para a desmineralização em apenas duas marcas estudadas. Em relação ao poder cariogênico, apenas uma das formulações analisadas apresentou a sacarose descrita de forma direta entre seus constituintes, apesar de os valores de SST se apresentarem relativamente elevados em quase todas as marcas. Ressalte-se que dose e frequência de uso são fatores importantes na análise dos potenciais de erosão dentária e do risco de desenvolvimento de cárie, devendo ser reforçada, com o paciente, a necessidade de manutenção de uma adequada higiene oral, o que também minimiza o surgimento dessas complicações.

\section{REFERÊNCIAS}

AIRES, C. P. et al. Effect of sucrose concentration on dental biofilm formed in situ and on enamel demineralization. Caries res., Basel, v. 40, n. 1 , p. 28-32, 2006

ASSOCIATION OF OFFICIAL ANALYTICAL CHEMISTS. Official Methods of Analysis. 14th ed. Arlington, 1984.

BALBANI, A. P. S.; STELZER, L.B.; MONTOVANI, J. C. Excipientes de medicamentos e informações da bula. Rev. bras. otorrinolaringol., Rio de Janeiro, v. 72, n. 3, p. 400-406, maio/ jun. 2006.

CAVALCANTI, A.L. et al. In vitro analysis of the cariogenic and erosive potential of pediatric antitussive liquid oral medications. Tanzan. j. health res., Tanzania, v.14, n.2, p. 1-8, Apr. 2012.

DUARTE, S. et al. Influences of starch and sucrose on streptococcus mutans biofilms. Oral. microbiol. Immunol., Copenhagen, v. 23, n. 3, p. 206-212, June 2008.

DURWARD, C.; THOU, T. Dental caries and sugar-containing liquid medicines for children in New Zeland. N. Z. dent. j., Dunedin, v. 93, n. 414, p. 124-129, Dec. 1997.

FERREIRA, A. O.; SOUZA, G.F. Preparações orais líquidas. Pharmabooks. 3. ed. São Paulo, 2005.

FISCHER D.J.; EPSTEIN J. B. Management of patients who have undergone head and neck cancer therapy. Dent. clin. North. Am., Philadelphia, v. 52, p.39-60, Jan. 2008.

MOREIRA, R. Dossiê acidulantes. Foods ingredientes Brasil, São Paulo, v.19, p. 26-30, 2011.

GORDÓN-NÚÑEZ, M. A.; PINTO L. P. Candidíase e sua relação com a mucosite oral em pacientes oncológicos pediátricos. Rev. bras. patol. oral, Natal, v. 2, n. 2, p. 4-9, abr./jun. 2003.

HAMILTON-MILLER, J. M. T. The effect of $\mathrm{pH}$ and temperature on the stability and bioactivity of nystatin and amphotericin B. J. pharm. pharmacol., London, n. 25, p. 401-407, May 1973.

LALLA, R.V. et al. A systematic review of oral fungal infections in patients receiving cancer therapy. Support. care cancer, Berlin, v.18, n.8, p.985992, Aug. 2010.

MARQUEZAN, M. et al. Medicamentos utilizados por pacientes odontopediátricos e seu potencial cariogênico. RPG, Rev. Pós-Grad., São Paulo, v.13, n. 4, p. $334-339$, out./dez. 2006.

MEURMAN, J. H. et al. Oral sources of septicemia in patients with malignancies. Oral oncol., Oxford, v.33, n.6, p.389-397, Nov. 1997.

PAPPAS, P. G. et al. Clinical practice guidelines for the management of candidiasis: 2009 update by the Infectious Diseases Society of America. Clin. Infect. Dis., Chicago, v. 48, p. 503-535, Mar. 2009.

PHILLIPS, S. M.; DELLINGER, T.M. Dental decay due to xerostomia and nystatin. Ann. pharmacother., Cincinnati, v. 39, p. 1758, Aug. 2005.

REDONDO, D.C.H.; MONTOYA, J. H. E. Complicaciones orales en pacientes 
sometidos a radioterapia: revisión de literature. Univ. odontol., Bogotá, v. 31, n. 67, p. 111-129, ul/dic. 2012.

SANTINHO, A. J. P.; WALDOW, C.; SANTOS, S. B dos. Estudo sobre a correlação do potencial cariogênico e do $\mathrm{pH}$ de xaropes pediátricos. Rev. bras. farm., Rio de Janeiro, v. 89, n.2, p.88-90, jan. 2008.

SOUSA, R. I. M. de et al. Potencial erosivo e cariogênico de anti-hista- mínicos de uso infantil. RFO UPF, Passo Fundo, v. 3, n.15, p. 255-260, set./dez. 2010.

VERMERIE, J. N. et al. Stability of nystatin in mouthrinses; effect of $\mathrm{pH}$, temperature, concentration and colloidal silver addition, studied using an in vitro antifungal activity. Pharm. world. sci., Dordrecht, v. 19, n.4, p. 197-201, Aug. 1997.

Submetido em: 19/11/2018

Aceito em: 29/11/2018 